\title{
Instrumentos para avaliação de feridas: scoping review
}

\author{
Instruments for wound assessment: scoping review \\ Instrumentos para la evaluación de heridas: revisión del alcance
}

Recebido: 09/08/2021 | Revisado: 24/08/2021 | Aceito: 24/08/2021 | Publicado: 25/08/2021

Camila Castanho Cardinelli

ORCID: https://orcid.org/0000-0003-1676-8324

Universidade Federal do Rio de Janeiro, Brasil

E-mail: camila.cardinelli@gmail.com

Luis Phillipe Nagem Lopes

ORCID: https://orcid.org/0000-0002-9186-110X Universidade Federal do Rio de Janeiro, Brasil

E-mail: luisphillipelopes@hotmail.com

Karina Chamma Di Piero

ORCID: https://orcid.org/0000-0002-0102-6785

Universidade Federal do Rio de Janeiro, Brasil

E-mail: kadipiero@gmail.com

Zaida Maria Faria de Freita

ORCID: https://orcid.org/0000-0001-6044-1711

Universidade Federal do Rio de Janeiro, Brasil E-mail: zaida@pharma.ufrj.br

\begin{abstract}
Resumo
Este estudo buscou identificar e descrever os principais instrumentos utilizados para análise de feridas, além de levantar sua validação e adaptação transcultural para a língua portuguesa. Trata-se de uma revisão de escopo elaborada segundo o método proposto pelo Joanna Briggs Institute. As bases de dados Portal Regional da Biblioteca Virtual de Saúde (BVS), MEDLINE (sob a interface do Pubmed), Scopus, Web of Science, CINAHL, LILACS e Scielo foram consultadas a fim de identificar estudos que utilizaram pelo menos um instrumento validado para a avaliação de feridas até janeiro de 2021. A literatura cinzenta foi verificada através do Google Scholar. A estratégia de busca foi desenvolvida utilizando Descritores em Saúde (DeCS) e termos MeSH como cicatrização, estudo de avaliação e avaliação em enfermagem. Os estudos foram incluídos independente do desenho utilizado, apenas estudos que não relatassem o uso de algum instrumento para avaliação de feridas, ou estudos envolvendo modelos pré-clínicos foram excluídos. Dois revisores selecionaram os artigos de forma independente. Os instrumentos encontrados foram descritos e categorizados após a calibração dos revisores. 51 instrumentos para avaliação de feridas foram encontrados em um total de 110 estudos selecionados. Esses instrumentos envolveram diferentes abordagens na prática clínica (cicatrização, infecção, dor provocada pela ferida, etc). No entanto, apenas oito (15,7\%) foram adaptados transculturalmente para utilização na língua portuguesa. Este estudo sugere que mais instrumentos sejam desenvolvidos e adaptados para a língua portuguesa do Brasil, a fim de que o diagnóstico e tratamento de feridas possam ser mais precisos.
\end{abstract}

Palavras-chave: Cicatrização; Ferimentos e lesões; Prática clínica baseada em evidências.

\begin{abstract}
The aim is to identify and describe the main instruments used for wound analysis, in addition to raising their validation and cross-cultural adaptation to the Portuguese language. This is a scope review prepared according to the method proposed by the Joanna Briggs Institute. The Regional Portal of the Virtual Health Library (BVS), MEDLINE (under the Pubmed interface), Scopus, Web of Science, CINAHL, LILACS and Scielo databases were consulted in order to identify studies that used at least one instrument validated for wound assessment by January 2021. Gray literature was verified using Google Scholar. The search strategy was developed using Health Science Descriptors (DeCS) and MeSH terms such as wound healing, assessment study and nursing assessment. Studies were included regardless of the design used, only studies that did not report the use of any instrument for wound assessment, or studies involving preclinical models were excluded. Two reviewers selected the articles independently. The instruments found were described and categorized after the reviewers' calibration. 51 instruments for wound assessment were found in a total of 110 selected studies. These instruments involved different approaches in clinical practice (wound healing, infection, pain caused by the wound, etc.). However, only eight $(15.7 \%)$ were crossculturally adapted for use in the Portuguese language. This study suggests that more instruments must be developed and adapted to the Portuguese language of Brazil, so that the diagnosis and treatment of wounds can be more accurate. Keywords: Wound healing; Wounds and injuries; Evidence-based practice.
\end{abstract}




\begin{abstract}
Resumen
Este estudio buscó identificar y describir los principales instrumentos utilizados para el análisis de heridas, además de elevar su validación y adaptación transcultural a la lengua portuguesa. Se trata de una revisión del alcance preparada según el método propuesto por el Instituto Joanna Briggs. Se consultaron las bases de datos del Portal Regional de la Biblioteca Virtual en Salud (BVS), MEDLINE (bajo la interfaz Pubmed), Scopus, Web of Science, CINAHL, LILACS y Scielo para identificar estudios que utilizaron al menos un instrumento validado para la evaluación de heridas por Enero de 2021. Se verificó la literatura gris utilizando Google Scholar. La estrategia de búsqueda se desarrolló utilizando descriptores de salud (DeCS) y términos MeSH como curación, estudio de evaluación y evaluación de enfermería. Los estudios se incluyeron independientemente del diseño utilizado, solo se excluyeron los estudios que no informaron el uso de ningún instrumento para la evaluación de heridas o los estudios que incluían modelos preclínicos. Dos revisores seleccionaron los artículos de forma independiente. Los instrumentos encontrados fueron descritos y categorizados después de la calibración de los revisores. Se encontraron 51 instrumentos para la evaluación de heridas en un total de 110 estudios seleccionados. Estos instrumentos implicaron diferentes enfoques en la práctica clínica (cicatrización, infección, dolor causado por la herida, etc.). Sin embargo, solo ocho (15,7\%) fueron adaptados transculturalmente para su uso en el idioma portugués. Este estudio sugiere que se desarrollen y adapten más instrumentos al idioma portugués de Brasil, para que el diagnóstico y tratamiento de las heridas sea más preciso.

Palabras clave: Cicatrización de heridas; Heridas y lesiones; Práctica clínica basada en la evidencia.
\end{abstract}

\title{
1. Introdução
}

Ferida pode ser definida como qualquer interrupção na continuidade de um tecido corpóreo, em maior ou menor extensão, causada, principalmente, por trauma ou desencadeada por uma afecção clínica. Elas são consideradas um problema de saúde pública, devido ao impacto psicológico, social e econômico para o paciente, com elevados e crescentes custos para o Sistema Único de Saúde (SUS) (Mandelbaum et al., 2003). Sendo assim, o cuidado de feridas é uma temática complexa que vem sendo cada vez mais estudada com vistas à melhoria da prática clínica com base em evidências científicas.

Na literatura, as terminologias utilizadas para descrever e avaliar lesões de pele são diversas; tal fato pode dificultar o estabelecimento de um consenso sobre quais parâmetros são mais apropriados para o monitoramento (Abbade, 2010).

Existem diversos instrumentos para avaliar feridos publicados na literatura científica internacional, mas grande parte ainda não passou pelo processo de adaptação transcultural e validação, situação que determina prejuízo na sua aplicação no Brasil. Ademais, dentre os instrumentos validados para a avaliação de feridas, alguns são direcionados para avaliação de lesões de etiologia específica, enquanto outros fazem uma abordagem mais geral (Garbuio et al., 2018).

Diretrizes e recomendações de saúde e associações científicas, além de várias revisões sistemáticas, incluindo revisões Cochrane, estão disponíveis para orientar a seleção da linha terapêutica mais indicada para tratamento de feridas (Bergin et al., 2012; Lipsky et al., 2012; Chadwick et al., 2013; International Diabetes Federation, 2015). No entanto, poucos estudos abordam a etapa que precede o tratamento propriamente dito, isto é, a avaliação dessas feridas (Tchero et al., 2019). Por isso, identificar e mapear instrumentos disponíveis para avaliação pode ser útil para informar profissionais de saúde sobre ferramentas disponíveis, bem como a disponibilidade desses instrumentos no Brasil.

Portanto, objetiva-se com este trabalho identificar e descrever os principais instrumentos utilizados para análise de feridas, além de levantar sua validação e adaptação transcultural para a língua portuguesa, situação que pode gerar recomendação da sua utilização na prática clínica no manejo de feridas.

\section{Metodologia}

Trata-se de uma revisão de escopo elaborada segundo o método proposto pelo Joanna Briggs Institute (Peters et al., 2020) visando explorar a literatura e mapear instrumentos disponíveis para avaliar feridas na prática clínica. Esta revisão foi conduzida seguindo as recomendações do checklist PRISMA-ScR (Tricco et al., 2018). O protocolo de revisão foi previamente registrado no Open Science Framework (Cardinelli et al., 2021). 


\section{Fonte de informações}

A busca dos artigos foi conduzida durante o mês de abril de 2021 nas bases de dados: Portal Regional da Biblioteca Virtual de Saúde (BVS), MEDLINE (sob a interface do Pubmed), Scopus, Web of Science, CINAHL, LILACS e Scielo, a fim de identificar estudos relevantes publicados.

\section{Outros recursos de busca}

Adicionalmente, uma pesquisa foi realizada no Google Scholar para a avaliação de literatura cinzenta com o objetivo de identificar estudos não indexados nas bases de dados acima mencionadas e as referências dos estudos incluídos também foram consultadas visando a identificação de estudos potencialmente elegíveis.

\section{Estratégia de Busca}

Uma estratégia de busca foi elaborada na base de dados Portal Regional da BVS e adaptada para as demais bases e incluiu termos Medical Subject Headings (MESH) ou Descritores em Saúde (DeCS) e palavras chaves relacionados à avaliação de feridas como cicatrização, cicatrização de feridas, cicatrização de ferimentos, estudo de avaliação, avaliação em enfermagem, protocolos de enfermagem; estes termos foram correlacionados a partir de operadores booleanos (OR e AND). A estratégia utilizada no portal regional da BVS foi: “(cicatrização) OR (cicatrização de feridas) OR (cicatrização de ferimentos) OR (wound healing) AND (estudo de avaliação) OR (estudos de avaliação) OR (evaluation study) OR (evaluation studies) AND (avaliação em enfermagem) AND (protocolos de enfermagem) OR (nursing assessment) OR (assessment, nursing) OR (nursing protocols) OR (protocols, nursing) AND (pressure ulcer scale for healing) OR (push)”. Esta mesma estratégia foi adaptada para as demais bases.

\section{Critérios de Elegibilidade}

Ambos os estudos primários e secundários foram analisados, independentemente de seu desenho de estudo ou status de publicação. Foram incluídos documentos relativos a qualquer tipo de estudo que tenha utilizado pelo menos uma ferramenta para a avaliação de feridas. Estudos envolvendo modelos pré-clínicos foram excluídos.

\section{Processo de seleção dos estudos}

A seleção dos títulos e resumos foi realizada por dois revisores independentes (CCC e LPNL) com base nos critérios de elegibilidade. Quaisquer discordâncias entre os revisores foram resolvidas por consenso.

\section{Extração de dados}

Os revisores foram calibrados extraindo pelo menos três artigos, e então foi realizado o consenso. Esse procedimento ocorreu até que os revisores conseguissem extrair os dados que identificavam. Enquanto ferramenta de coleta de dados foi

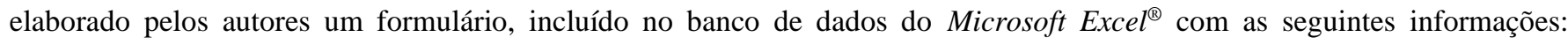
autor/ano, nome do instrumento, etiologia de ferida avaliada pelo instrumento, objetivo de avaliação do instrumento para prática clínica e se este instrumento citado foi ou não adaptado/validado transculturalmente para uso na língua portuguesa. Todas estas variáveis foram analisadas frente ao escopo da revisão realizada.

\section{Resultados}

O levantamento dos estudos nas bases de dados ocorreu conforme fluxograma da identificação, seleção e inclusão dos estudos - PRISMA (Figura 1). A busca inicial para este estudo recuperou 1855 estudos, dos quais 1253 foram retirados durante 
a triagem de títulos e resumos e 6 estudos foram excluídos após a leitura do texto na íntegra. Sessenta e um documentos foram encontrados por busca manual em outros recursos de busca. A seleção final incluiu 110 documentos, os quais foram analisados em seu texto completo conforme os objetivos da revisão.

Figura 1: Fluxograma de identificação, seleção e inclusão dos estudos - PRISMA.

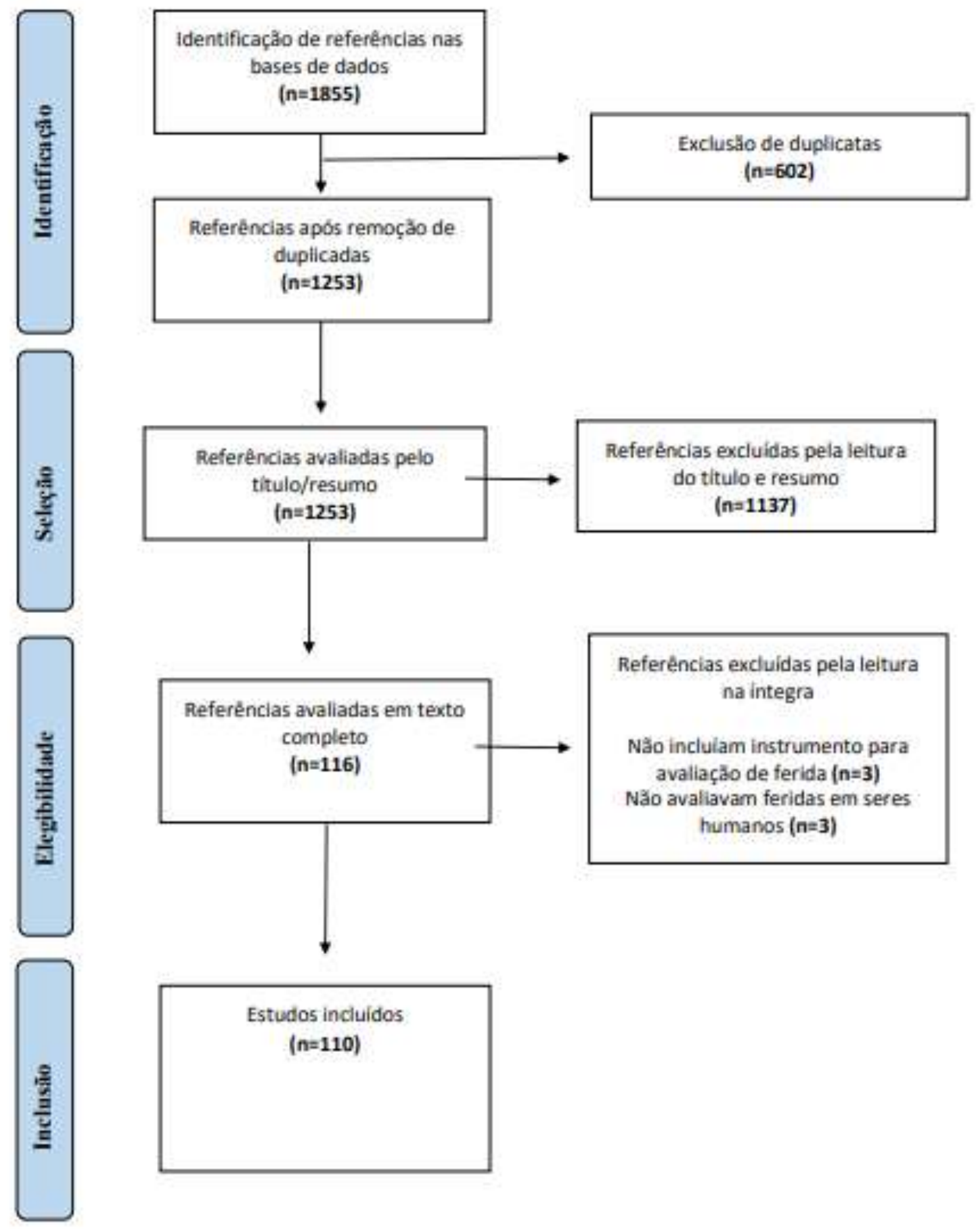

Fonte: Cardinelli et al. (2021). 
Ainda que nenhum filtro de tempo tenha sido utilizado no processo de busca dos artigos, a dimensão temporal desta revisão compreendeu o período de 1986 a 2019 (Figura 2) e apontou um crescimento ao longo do tempo, especialmente a partir dos anos 2000 das publicações referentes à temática.

Figura 2: Dimensão temporal da revisão por ano de publicação (1986 - 2019).

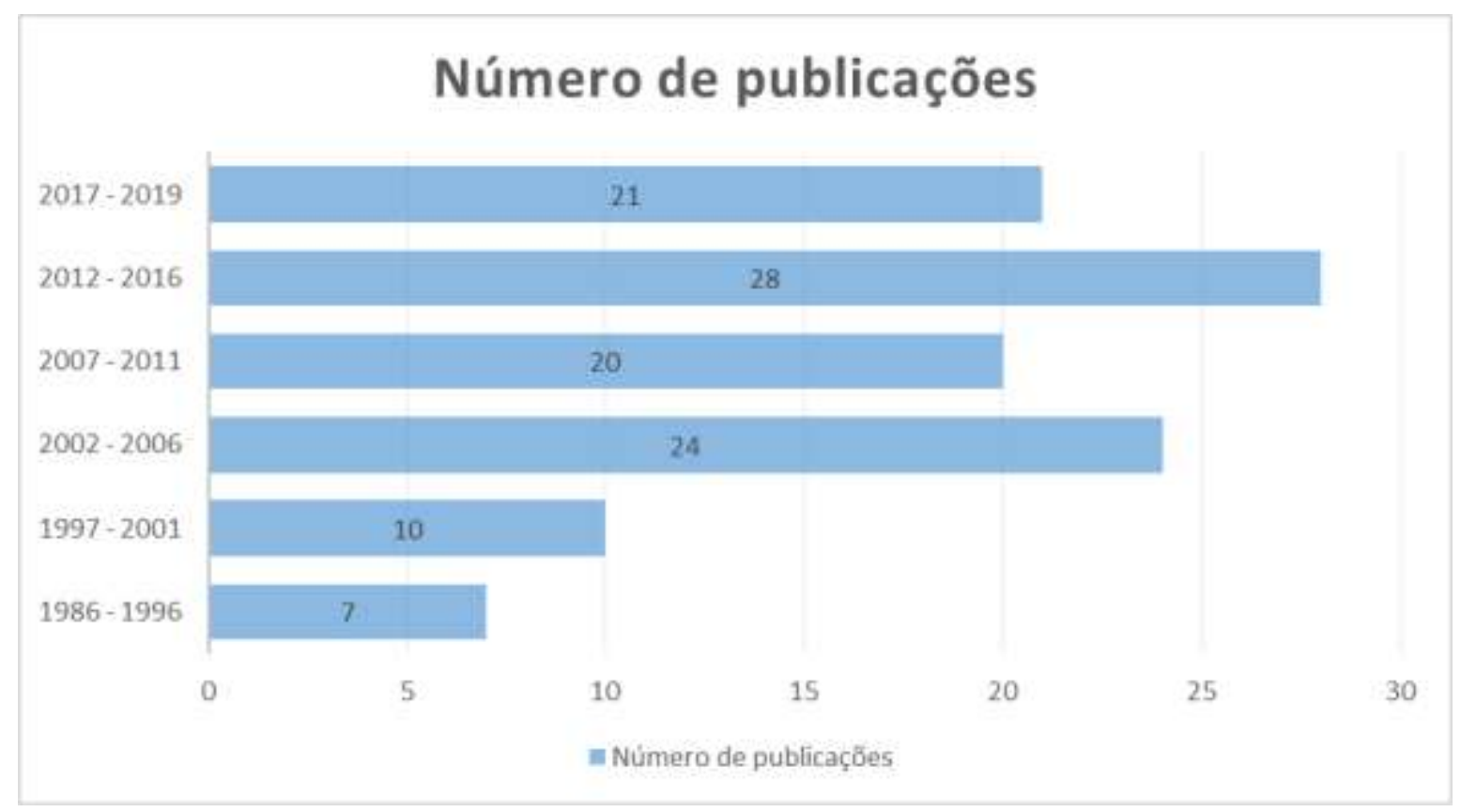

Fonte: Cardinelli et al. (2021).

Sobre os instrumentos levantados nos estudos analisados, foram encontrados 51 para a avaliação de feridas (Tabela 1). Destes, apenas oito $(15,7 \%)$ foram adaptados transculturalmente para utilização na língua portuguesa. 
Tabela 1: Instrumentos para avaliação de feridas, levantados nos estudos analisados.

\begin{tabular}{|c|c|c|c|c|}
\hline $\begin{array}{l}\text { Nome do } \\
\text { instrumento }\end{array}$ & Autores/ ano de publicação & $\begin{array}{c}\text { Descrição do } \\
\text { instrumento/ etiologia } \\
\text { avaliada }\end{array}$ & $\begin{array}{l}\text { Adaptação para } \\
\text { português/ } \\
\text { validação }\end{array}$ & $\begin{array}{l}\text { Recomendação } \\
\text { para prática } \\
\text { clínica }\end{array}$ \\
\hline $\begin{array}{l}\text { Pressure Ulcer } \\
\text { Scale For } \\
\text { Healing (PUSH } \\
\text { TOOL) }\end{array}$ & $\begin{array}{l}\text { Arisandi et al., 2016; Berlowitz et al., } \\
\text { 2005;Carbonari et al., 2013; Choi et al., 2016; } \\
\text { Chuangsuwanich et al., 2013; Desneves et al., 2005; } \\
\text { Espírito Santo et al., 2013; Flanagan, 2003; Gardner } \\
\text { et al., 2011; Gardner et al., 2005; George-Saintilus et } \\
\text { al., 2009; Gonzalez, 2017; Greatrex-White; Moxey, } \\
\text { 2015; Oseni \& Adejumo, 2014; Günes, 2009; Günes } \\
\text { \& Eser, 2007;Hon et al., 2010;Kim et al., 2014; Lee } \\
\text { et al., 2006; Magnoni et al., 2013; Milne et al., 2012; } \\
\text { Palagi et al., 2015; Panfil \& Line, 2007; Ratliff, } \\
\text { 2018; Ratliff; Rodeheaver, 2005; Santos et al., 2005; } \\
\text { Santos et al., 2007; Shepherd \& Nixon, 2013; Stotts } \\
\text { et al., 2001; Sung \& Park, 2011; Thomason et al., } \\
\text { 2014; Woodbury et al., 1999; Yapucu-Günes \& } \\
\text { Eser, 2007; Young et al., 2017; Zhang et al., 2014 }\end{array}$ & $\begin{array}{c}\text { Lesão por pressão } \\
\text { Úlceras diabéticas } \\
\text { Úlceras venosas } \\
\text { Úlceras arteriais } \\
\text { Feridas agudas } \\
\text { Feridas crônicas }\end{array}$ & Sim & Cicatrização \\
\hline TIME & $\begin{array}{l}\text { Atkin, 2019; Ayello et al., 2004; Bullough \& } \\
\text { Spruce, 2013; Cook, 2011; Dowsett \& Hall, 2019; } \\
\text { Irgens et al., 2019; Greatrex-White \& Moxey, 2015; } \\
\text { Jones et al., 2018; Lo et al., 2007; Ousey et al., } \\
\text { 2016; Vowden, 2005 }\end{array}$ & Todas as etiologias & Sim & $\begin{array}{l}\text { Preparo do leito } \\
\text { da lesão }\end{array}$ \\
\hline $\begin{array}{l}\text { Bates-Jensen } \\
\text { Wound } \\
\text { Assessment Tool }\end{array}$ & $\begin{array}{l}\text { Arisandi et al., 2016; Glik et al., 2015; Greatrex- } \\
\text { White \& Moxey, 2015; Harris et al., 2018; Karahan } \\
\text { et al., 2018; Tao et al., 2015; Thomason et al., 2014; } \\
\text { Vitsos et al., } 2019\end{array}$ & $\begin{array}{c}\text { Queimaduras } \\
\text { Lesões por pressão } \\
\text { Ferida operatória } \\
\text { Úlceras venosas } \\
\text { Feridas agudas e } \\
\text { crônicas Feridas } \\
\text { infectadas }\end{array}$ & Sim & Cicatrização \\
\hline $\begin{array}{l}\text { Leg Ulcer } \\
\text { Mensuring Tool } \\
\text { (LUMT) }\end{array}$ & $\begin{array}{l}\text { Greatrex-White \& Moxey, 2015; Panfil \& Linde, } \\
\text { 2007; Woodbury et al., 2004; Silveira et al., } 2020\end{array}$ & $\begin{array}{l}\text { Úlceras venosas, } \\
\text { arteriais e diabéticas }\end{array}$ & Sim & $\begin{array}{l}\text { Avaliação de } \\
\text { cicatrização/Dor }\end{array}$ \\
\hline RESVECH 2.0 & Domingues et al., 2018 & Feridas crônicas & Sim & Cicatrização \\
\hline $\begin{array}{l}\text { ISTAP Skin } \\
\text { Tear } \\
\text { Classification }\end{array}$ & Silva et al., 2018 & Skin Tears & Sim & Classificação \\
\hline $\begin{array}{l}\text { STAR Skin Tear } \\
\text { Classification } \\
\text { System }\end{array}$ & Strazzieri-Pulido et al., 2015 & Skin Tears & Sim & Classificação \\
\hline $\begin{array}{l}\text { Pressure Sore } \\
\text { Score Tool } \\
\text { (PSST) }\end{array}$ & $\begin{array}{l}\text { Bates-Jensen et al., 1992; Bates-Jensen, 1997; } \\
\text { Bates-Jensen \& Mcnees, 1996; Bolton et al., 2004; } \\
\text { Flanagan, 2003; Motta et al., 1999; Mutluoglu et al., } \\
\text { 2016; Pak et al., 2018; Panfil \& Linde, 2007; Sanada } \\
\text { et al., 2004; Whitney et al., 2001; Woodbury et al., } \\
1999\end{array}$ & $\begin{array}{c}\text { Lesões por pressão } \\
\text { Úlceras neuropáticas } \\
\text { Ferida operatória } \\
\text { Úlceras venosas } \\
\text { Feridas agudas e } \\
\text { crônicas } \\
\text { Feridas infectadas }\end{array}$ & Sim & Cicatrização \\
\hline $\begin{array}{l}\text { Digital Ulcers } \\
\text { Score (DUS) }\end{array}$ & Ahrens et al., 2016 & Úlceras digitais & Não & Classificação \\
\hline REEDA & Alvarenga et al., 2015 & Episiotomia & Não & Cicatrização \\
\hline $\begin{array}{l}\text { Diabetic Foot } \\
\text { Ulcer } \\
\text { Assessment } \\
\text { Scale (DFUAS) }\end{array}$ & Arisandi et al., 2016 & Úlceras diabéticas & Não & Cicatrização \\
\hline
\end{tabular}


Research, Society and Development, v. 10, n. 11, e144101119246, 2021

(CC BY 4.0) | ISSN 2525-3409 | DOI: http://dx.doi.org/10.33448/rsd-v10i11.19246

\begin{tabular}{|c|c|c|c|c|}
\hline $\begin{array}{c}\text { Barber } \\
\text { Measuring Tool }\end{array}$ & Barber, 2008 & Todas as etiologias & Não & Cicatrização \\
\hline ASEPSIS & $\begin{array}{l}\text { Byrne et al., 1988; Copanitsanou et al., 2018; } \\
\text { Koschwane \& Broadbent, 2011; Haraden \& } \\
\text { Jaenicke, 2006; Heiner et al., 2002; Melling \& } \\
\text { Leaper, 2006; Mphande et al., 2007; Totty et al., } \\
\text { 2019; Wilson et al., } 1986\end{array}$ & $\begin{array}{l}\text { Feridas infectadas } \\
\text { Feridas operatórias }\end{array}$ & Não & Infecção \\
\hline $\begin{array}{l}\text { Wound Trend } \\
\text { Scale (WTS) }\end{array}$ & Campbell et al., 2016 & $\begin{array}{l}\text { Úlceras diabéticas, } \\
\text { arteriais e venosas }\end{array}$ & Não & Cicatrização \\
\hline $\begin{array}{l}\text { Southampton } \\
\text { wound } \\
\text { assessment scale } \\
\text { (SWAS) }\end{array}$ & Claroni et al., 2016; Koschwane \& Broadbent, 2011 & Ferida operatória & Não & Infecção \\
\hline DESIGN & $\begin{array}{l}\text { Hisashige \& Ohura, 2012; Nagase et al., 2013; } \\
\text { Nakagami et al., 2010; Omote et al., 2005; Panfil \& } \\
\text { Linde, 2007; Sanada et al., 2010; Sanada et al., } \\
\text { 2004; Takahashi et al., } 2006\end{array}$ & Lesões por pressão & Não & Cicatrização \\
\hline $\begin{array}{c}\text { The } \\
\text { Granulometer } \\
\text { Scale }\end{array}$ & Cohen et al., 1998 & Granulomas & Não & Cicatrização \\
\hline $\begin{array}{l}\text { The McGill Pain } \\
\text { Questionnaire }\end{array}$ & De Laat et al., 2005 & Lesão por pressão & Não & Dor \\
\hline $\begin{array}{l}\text { Leg Ulcer } \\
\text { Assessment Tool } \\
\quad \text { (LUAT) }\end{array}$ & Dereure et al., 2005; Vandenkerkhof et al., 2013 & $\begin{array}{l}\text { Úlceras venosas, } \\
\text { arteriais e diabéticas }\end{array}$ & Não & Cicatrização \\
\hline $\begin{array}{l}\text { Peri-ulcer skin } \\
\text { assessment scale }\end{array}$ & Dereure et al., 2005 & Úlceras venosas & Não & $\begin{array}{l}\text { Avaliação de } \\
\quad \text { Risco }\end{array}$ \\
\hline $\begin{array}{l}\text { Wound Bed } \\
\text { Score System } \\
\text { (WBS) }\end{array}$ & Falanga et al., 2006; Milne et al., 2012 & Úlceras venosas & Não & $\begin{array}{l}\text { Preparo do leito } \\
\text { da lesão }\end{array}$ \\
\hline Sessing Scale & $\begin{array}{l}\text { Ferrel et al., 1995; Panfil \& Linde, 2007; Woodbury } \\
\text { et al., 1999; Greatrex-White \& Moxey, 2015; }\end{array}$ & Lesão por pressão & Não & Cicatrização \\
\hline $\begin{array}{l}\text { Clinical signs } \\
\text { and symptoms } \\
\text { checklist }\end{array}$ & $\begin{array}{l}\text { Gardner et al., 2001a; Gardner et al., 2007; Gardner } \\
\text { et al., 2001b }\end{array}$ & $\begin{array}{l}\text { Úlceras diabéticas } \\
\text { Feridas infectadas }\end{array}$ & Não & Infecção \\
\hline $\begin{array}{c}\text { Wound } \\
\text { Assessment } \\
\text { Inventory (WAI) }\end{array}$ & Koschwane \& Broadbent, 2011 & $\begin{array}{l}\text { Feridas infectadas } \\
\text { Feridas operatórias }\end{array}$ & Não & $\begin{array}{l}\text { Cicatrização/Infla } \\
\text { mação }\end{array}$ \\
\hline $\begin{array}{l}\text { The Wound } \\
\text { Healing Self } \\
\text { Score }\end{array}$ & Haraden \& Jaenicke, 2006; Reeves et al., 2017 & Ferida operatória & Não & Cicatrização \\
\hline $\begin{array}{l}\text { S(AD)SAD } \\
\text { system }\end{array}$ & Jeffcoate et al., 2006; Kasbekar et al., 2017 & Úlceras diabéticas & Não & $\begin{array}{l}\text { Cicatrização/Infe } \\
\text { cção }\end{array}$ \\
\hline $\begin{array}{l}\text { The Healing } \\
\text { Scale }\end{array}$ & Johnson \& Miller, 1996 & Úlceras venosas & Não & Cicatrização \\
\hline $\begin{array}{l}\text { The Johnson } \\
\text { Scale }\end{array}$ & Johnson \& Miller, 1996 & Úlceras venosas & Não & Cicatrização \\
\hline $\begin{array}{c}\text { PEDIS } \\
\text { classification }\end{array}$ & Kasbekar et al., 2017 & Úlceras diabéticas & Não & $\begin{array}{l}\text { Complicações da } \\
\text { ferida }\end{array}$ \\
\hline MEASURE & Keast et al., 2004 & Todas as etiologias & Não & Cicatrização \\
\hline SMArt Wound & Kooner et al., 2018 & Feria operatória & Não & Cicatrização \\
\hline
\end{tabular}


Research, Society and Development, v. 10, n. 11, e144101119246, 2021

(CC BY 4.0) | ISSN 2525-3409 | DOI: http://dx.doi.org/10.33448/rsd-v10i11.19246

\begin{tabular}{|c|c|c|c|c|}
\hline Tool & & & & \\
\hline $\begin{array}{l}\text { Triangle of } \\
\text { Wound } \\
\text { Assessment }\end{array}$ & Lázaro-Martínez et al., 2018 & Feridas crônicas & Não & $\begin{array}{l}\text { Avaliação de } \\
\text { Risco/ } \\
\text { Cicatrização }\end{array}$ \\
\hline $\begin{array}{l}\text { Bluebelle } \\
\text { Wound Healing } \\
\text { Questionnaire }\end{array}$ & Macefield, 2018; Macefield et al., 2019 & $\begin{array}{l}\text { Feridas infectadas } \\
\text { Feridas operatórias }\end{array}$ & Não & $\begin{array}{l}\text { Avaliação de } \\
\quad \text { Risco }\end{array}$ \\
\hline DIAFORA & $\begin{array}{l}\text { Monteiro-Soares \& Dinis-Ribeiro, 2016; Kasbekar } \\
\text { et al., } 2017\end{array}$ & Úlceras diabéticas & Não & $\begin{array}{l}\text { Avaliação de } \\
\text { Risco }\end{array}$ \\
\hline $\begin{array}{l}\text { TIME clinical } \\
\text { decision support } \\
\text { tool (CDST) }\end{array}$ & Moore et al., 2019 & Todas as etiologias & Não & $\begin{array}{l}\text { Preparo do leito } \\
\text { da lesão }\end{array}$ \\
\hline $\begin{array}{l}\text { Applied Wound } \\
\text { Management } \\
\text { Tool }\end{array}$ & Murphy, 2009; Greatrex-White \& Moxey, 2015; & Todas as etiologias & Não & Cicatrização \\
\hline $\begin{array}{l}\text { Photographic } \\
\text { Wound } \\
\text { Assessment Tool } \\
\text { (PWAT) }\end{array}$ & $\begin{array}{l}\text { Mutluoglu et al., 2016; Panfil \& Linde, 2007; } \\
\text { Houghton et al., } 2000\end{array}$ & Todas as etiologias & Não & $\begin{array}{l}\text { Documentação de } \\
\text { ferida }\end{array}$ \\
\hline $\begin{array}{l}\text { PUHP-Ohura } \\
\text { wound- } \\
\text { assessment tool }\end{array}$ & Ohura et al., 2011; Panfil \& Linde, 2007 & Lesão por pressão & Não & Cicatrização \\
\hline $\begin{array}{c}\text { ABDEFS' } \\
\text { scoring system }\end{array}$ & Oluwatosin et al., 1998 & Ferida Crônica & Não & Cicatrização \\
\hline CODED & Panfil \& Linde, 2007 & Todas as etiologias & Não & Cicatrização \\
\hline DESIGN-R & Sanada et al., 2011; Zhong et al., 2013 & Lesões por pressão & Não & Cicatrização \\
\hline $\begin{array}{l}\text { Yarkony-Kirk } \\
\text { scale }\end{array}$ & Sharp, 2004 & Lesão por pressão & Não & $\begin{array}{l}\text { Avaliação de } \\
\quad \text { Risco }\end{array}$ \\
\hline $\begin{array}{l}\text { Wound Healing } \\
\text { Assessment and } \\
\text { Management } \\
\text { Tool (WHAM) }\end{array}$ & Shepherd \& Nixon, 2013 & $\begin{array}{l}\text { Úlceras venosas } \\
\text { Feridas cavitarias } \\
\text { Lesão por pressão } \\
\text { Todo as etiologias }\end{array}$ & Não & Cicatrização \\
\hline $\begin{array}{l}\text { Wound Infection } \\
\text { Risk-Assessment } \\
\text { and Evaluation } \\
\text { tool (WIRE) }\end{array}$ & Siaw-Sakyi, 2017 & Feridas Infectadas & Não & $\begin{array}{l}\text { Infecção } \\
\text { Avaliação de } \\
\quad \text { Risco }\end{array}$ \\
\hline $\begin{array}{l}\text { SCI Pressure } \\
\text { Ulcer } \\
\text { Monitoring Tool } \\
\text { (SCI-PUMT) }\end{array}$ & Thomason et al., 2014 & Lesão por pressão & Não & Cicatrização \\
\hline $\begin{array}{c}\text { Healing } \\
\text { Progression } \\
\text { Rate (HPR) }\end{array}$ & Young et al., 2017 & Lesão por pressão & Não & Cicatrização \\
\hline $\begin{array}{l}\text { Sussman Wound } \\
\text { Healing Tool } \\
\text { (SWHT) }\end{array}$ & $\begin{array}{l}\text { Woodbury et al., 1999; Greatrex-White \& Moxey, } \\
\text { 2015; }\end{array}$ & Lesão por pressão & Não & Cicatrização \\
\hline $\begin{array}{l}\text { Wound Healing } \\
\text { Scale (WHS) }\end{array}$ & Woodbury et al., 1999 & Lesão por pressão & Não & Cicatrização \\
\hline $\begin{array}{l}\text { National Wound } \\
\text { Assessment } \\
\text { Form (NWAF) }\end{array}$ & Greatrex-White \& Moxey, 2015; & Todos as etiologias & Não & Cicatrização \\
\hline
\end{tabular}




\begin{tabular}{|c|l|c|c|c|}
\hline $\begin{array}{c}\text { NATVNS } \\
\text { Assessment } \\
\text { Chart for } \\
\text { Wound } \\
\text { management }\end{array}$ & Greatrex-White \& Moxey, 2015; & Todos as etiologias & Não & $\begin{array}{c}\text { Preparo do leito } \\
\text { da ferida } \\
\text { Documentação/Ci } \\
\text { catrização }\end{array}$ \\
\hline $\begin{array}{c}\text { Wound } \\
\text { Assessment and } \\
\text { Management } \\
\text { System (WAMS) }\end{array}$ & Greatrex-White \& Moxey, 2015; & & Não & $\begin{array}{c}\text { Preparo do leito } \\
\text { da ferida/ } \\
\text { Avaliação da } \\
\text { cicatrização } \\
\text { Escolha } \\
\text { Terapêutica }\end{array}$ \\
\hline
\end{tabular}

Fonte: Cardinelli et al. (2021).

\section{Discussão}

A prática da enfermagem especializada e qualificada é decisiva para a assistência, devendo ser avaliada a partir da construção e utilização de instrumentos de medida que favoreçam a caracterização do conhecimento teórico e prático pelo enfermeiro no cuidado ao paciente com ferida (Yamada \& Santos, 2009). Apesar do vasto número de instrumentos encontrados nesta busca, apenas oito foram adaptados transculturalmente para a língua portuguesa. Tal fato pode refletir ainda, a pouca valorização do seu uso na prática clínica.

Vale lembrar, que o cuidado de feridas realizado pelo enfermeiro necessita de bases científicas para sua prática assistencial, tendo em vista sua complexidade. As feridas determinam prejuízo físico ao indivíduo, podendo gerar incapacidades e até comprometer sua qualidade de vida, impactando negativamente o serviço de saúde devido altos gastos em tecnologias para o cuidado (Waidman et al., 2011).

A ferida pode ser avaliada e ter diferentes registros, podendo gerar interpretações divergentes ou conflitantes, se o enfermeiro não for especializado ou qualificado quanto à interpretação dos sinais e sintomas do paciente. Neste contexto, para garantir a confiança entre os observadores, independentemente de sua especialização e qualificação, faz-se necessário uso de instrumentos, com padrões e critérios de análise definidos para interpretação consensual (Healey, 1997).

Dentre as escalas encontradas neste estudo, aquelas que foram validadas e adaptadas transculturalmente para a língua portuguesa foram: Pressure Sore Status Tool (PSST), Bates Jensen Wound Assessment Tool (BWAT), a Pressure Ulcer Score for Healing - PUSH Tool (Escore de cicatrização de lesão por pressão), o acrônimo TIME, Leg Ulcer Mensuring Tool LUMT (Instrumento de mensuração de úlceras de perna), o RESVECH 2.0, o ISTAP Skin Tear Classification (Classificação ISTAP de Skin Tears) e o STAR Skin Tear Classification System (Sistema STAR de classificação de Skin Tears).

É possível compreender que a grande maioria dos instrumentos já existentes têm como foco a avaliação do processo de cicatrização e a instituição de tratamento. Ainda assim, existe a necessidade de investir em instrumentos que favoreçam a avaliação do diagnóstico de etiologias para melhor manejo das feridas.

A precursora das escalas, desenvolvida nos Estados Unidos em 1990, é a Pressure Sore Status Tool (PSST). É uma escala tipo likert para avaliação de cicatrização de lesões, composta de 13 variáveis com pontuação variando de 13 (cicatrização) a 65 (degeneração da ferida). As variáveis analisadas na escala são: tamanho, profundidade, bordas, descolamento, tipo e quantidade de tecido necrótico, tipo e quantidade de exsudato, edema e endurecimento perilesional, coloração da pele perilesional, tecido de granulação e epitelização (Bates-Jensen et al., 1992).

Em 2001, a escala foi atualizada e renomeada para Bates-Jensen Wound Assessment Tool (BWAT) visando ampliar o escopo de etiologias que a escala poderia ser utilizada além de lesões por pressão. Suas pontuações dos itens descolamento, tipo e quantidade exsudato, edema e endurecimento também foram modificadas. Somente 14 anos depois, em 2015, houve validação e adaptação para o português (Harris et al., 2010; Alves et al., 2015). 
Sua sucessora, a escala mais utilizada e conhecida, é a Pressure Ulcer Score for Healing, a PUSH Tool. Ela foi desenvolvida em 1996 por um time de especialistas em lesões por pressão (NPUAP) para ser instrumento de avaliação da cicatrização destas lesões e foi validada para português em 2005. É norteada por três parâmetros principais: área da ferida (escore de 0 a 10), quantidade de exsudato (escore de 0 a 3 ) e a aparência do leito da ferida (escore de 0 a 4). Sua proposta inicial era utilização exclusivamente em lesões por pressão, mas já existem estudos que aumentam este escopo para outras etiologias (Santos et al., 2005; Günes, 2009).

Já o TIME é um acrônimo instituído por um grupo de especialistas em 2003 e posteriormente revisado e publicado em 2004. Ele aborda uma ordem de observação clínica para o preparo do leito da lesão. A letra T - avalia tipo de tecido (viável ou não), I é infecção e inflamação, M (de moisture) avalia exsudato e o E (de edge) é a avaliação de bordas. Pode ser utilizado em qualquer tipo de ferida para instituição de tratamento, independente da fase de cicatrização (Aron \& Gamba, 2009; Ousey et al., 2016).

Em 2004, a escala Leg Ulcer Mensuring Tool (LUMT) foi desenvolvida no Canadá para avaliação de úlceras de membros inferiores de diversas etiologias. Engloba avalição clínica da ferida, dor e qualidade de vida. É composta por dois domínios, o domínio A respondido pelo profissional que avalia clinicamente a ferida em 14 itens numerados de 0 a 4 ; e o domínio B composto por três itens a serem respondidos pelo próprio paciente. É a escala adaptada mais recentemente, quase 16 anos depois, em 2020 (Silveira et al., 2020; Woodbury et al., 2004).

Em 2007, um painel de especialistas Skin Tear Audition Research (STAR) criou o STAR Skin Tear Classification System. Este instrumento avalia a ausência ou presença/viabilidade de retalho. Ele funciona como classificação, guia de tratamento e glossário para avaliações de lesões do tipo skin tears. É composto por cinco categorias de skin tears, sua respectiva descrição e fotografia para facilitar a identificação. Em 2015, foi validado e passou a se chamar "Sistema de Classificação STAR - Lesão por Fricção" e introduziu o termo "lesão por fricção" como tradução do termo. (Carville et al., 2007; Strazzieri-Pulido et al., 2015).

Em 2011, outro painel de especialista em skin tears, o International Skin Tear Advisory Panel (ISTAP) desenvolveu uma atualização com intuito de facilitar o uso da classificação anterior. O ISTAP Skin Tear Classification foi adaptado para português em 2018 e é composto por três categorias que igualmente avaliam ausência/presença de retalho de pele e sua visibilidade: 1) ruptura linear com retalho totalmente presente, 2) perda parcial do retalho e 3) perda total do retalho (Leblanc et al., 2013; Silva et al., 2018).

Por fim, também em 2011 na Espanha, foi desenvolvido um instrumento com enfoque avaliação do processo de cicatrização de feridas crônicas. O RESVECH 2.0 (Resultados Esperados da Avaliação da Cicatrização de Feridas Crônicas) é composto por seis variáveis de avaliação: dimensão, profundidade, bordas, tipos de tecido, exsudato e infecção/inflamação e resulta em um escore que varia de zero (cicatrização) a 35 (degeneração). Dentre essas, somente o subitem infecção/inflamação não pontua (Domingues et al., 2018; Restrepo-Medrano \& Verdú Soriano, 2011).

Um dos achados mais importantes desta revisão de escopo está relacionado ao número reduzido de instrumentos adaptados e validados para o português, sugerindo que, apesar de sua importância, estes instrumentos ainda são pouco utilizados na assistência. Na literatura brasileira, são escassos os estudos de utilização de instrumentos de medida baseados em situações da realidade, no contexto de feridas (Costa et al., 2015).

Isto reitera a importância da adaptação e validação transcultural de instrumentos para o Brasil, além do seu treinamento e prática clínica rotineira pela equipe de saúde com o objetivo de uma assistência baseada em evidências científicas. Vale lembrar, que o aumento dos estudos sobre a temática na década vigente pode demonstrar acentuação do interesse pelo uso de instrumentos com evidências de validação e adaptação transcultural para a realização de um manejo mais científico das feridas na prática clínica. 
Os instrumentos para avaliação de feridas são ferramentas importantes tanto na tomada de decisão diagnóstica quanto na preventiva e terapêutica, inclusive na realização do procedimento mais adequado, pois é capaz de nortear as melhores práticas para a recuperação do paciente com feridas (Dantas et al., 2012).

É importante considerar, que o desenvolvimento de lesões pode elevar taxas de morbimortalidade, aumento de custos globais no tratamento (insumos e recursos humanos) e acarretar ainda no aumento do tempo de hospitalização, e riscos adicionais como infecção hospitalar. Logo, torna-se mandatório que os profissionais de saúde sejam orientados e instrumentalizados para o diagnóstico correto e manejo mais adequado das feridas (Lima et al., 2017). Sobre as etiologias de feridas referidas nos instrumentos analisados, a mais citada foi a lesão por pressão, indicador de qualidade e segurança do cuidado em saúde.

As lesões por pressão ocorrem principalmente em áreas de proeminências ósseas e são decorrentes de pressão ou da combinação de pressão e fricção. Um dos fatores de risco que mais favorecem seu aparecimento é o peso do corpo contra a superfície de apoio (cama ou cadeira), causando pressão e comprometimento circulatório tecidual, que pode ser ainda associado a outros fatores externos de risco como, fricção, cisalhamento e alteração do microclima da pele em pacientes com déficit de mobilidade/atividade (EPUAP et al., 2019).

Conforme dados do Departamento de Informática do Sistema Único de Saúde (DATASUS), entre 2015 e 2019, as lesões por pressão (CID 10 - L89 úlcera de decúbito) foram a causa da morte de 1.029 pessoas no município do Rio de Janeiro (Brasil, 2021) refletindo sua importância diagnóstica e terapêutica com o objetivo de prevenção de mortes decorrentes de uma lesão que pode ser prevenida na prática clínica pela equipe de saúde.

A segunda etiologia mais identificada nos instrumentos de avaliação de feridas analisados foram úlceras venosas, que acometem os membros inferiores, normalmente secundárias à hipertensão venosa e insuficiência venosa crônica. Sua taxa de incidência varia de 10 a 35\% na população americana e essa prevalência pode aumentar em 4\% nos pacientes maiores de 65 anos (Evans et al., 2019).

E as feridas cirúrgicas ou operatórias, decorrentes de incisão intencional, podendo cicatrizar por primeira intenção, quando há rafia de bordas; segunda intenção, quando aberta evolui de forma orgânica pela progressão das fases de cicatrização mediante proliferação celular e neoformação tecidual e terceira intenção quando a ferida fica aberta por um tempo e sua reaproximação de bordas ocorre posteriormente em centro cirúrgico (Olczyk et al., 2014).

Este estudo é o primeiro a mapear e descrever ferramentas para avaliação de feridas, bem como categorizar quais instrumentos já estão validados no Brasil. Como limitação, destaca-se a impossibilidade de contato com alguns autores devido ausência de publicação na íntegra de alguns estudos, bem como teses e dissertações. Isso impossibilitou a ampliação da análise. Apesar de tais limitações, esta revisão de escopo contribui para a sistematização das evidências disponíveis sobre instrumentos para avaliação de feridas. Isso pode facilitar o acesso à informação das principais ferramentas disponíveis aos profissionais de saúde, além de ser útil para que futuros estudos sejam desenvolvidos para realizarem a adaptação transcultural de instrumentos ainda não validados no Brasil.

\section{Conclusão}

Esta revisão mapeou na literatura os instrumentos disponíveis para a avaliação de feridas, seus objetivos e quais destes instrumentos foram validados e adaptados transcultural para língua portuguesa, no Brasil. E diante disso, foi possível observar quanto ainda é pouco científica a avaliação de pacientes com feridas, desde o diagnóstico ao manejo clínico, situação que deve impactar negativamente na qualidade e segurança da assistência em saúde prestada no Brasil. 
Conclui-se que os instrumentos se utilizam de etiologias de feridas específicas para a elaboração de instrumentos que vão desde a confirmação diagnóstica, manejo preventivo até terapêutico, tudo com base científica a fim de determinar uma prática consensual, que auxilia a segurança e qualidade do cuidado prestado.

Diante disso, é relevante para a prática de enfermagem a elaboração ou adaptação/validação de instrumentos para contribuir na avaliação e manejo sistematizado de feridas com base no preceito da sistematização da assistência em enfermagem (SAE) que norteia o enfermeiro e a enfermagem com base na prática baseada em evidência científica.

\section{Referências}

Abbade, L. P. F. (2010). Preparo do leito da ferida. In W. Malagutti (Ed.), Curativos, Estomias e Dermatologia: uma abordagem profissional. (pp. 63-76). Martinari.

Alves, D. F. dos S., Almeida, A. O. de, Silva, J. L. G., Morais, F. I., Dantas, S. R. P. E., \& Alexandre, N. M. C. (2015). Translation and Adaptation of the Bates-Jensen Wound Assessment Tool for the Brazilian culture. Texto Contexto Enferm, 24(3), 826-833. https://doi.org/10.1590/0104-07072015001990014

Aron, S., \& Gamba, M. A. (2009). Preparo do Leito da Ferida e a História do TIMEWound Bed Preparation and a History of TIME. ESTIMA, Braz. J. Enterostomal Ther., 7(4).

Bates-Jensen, B. M., Vredevoe, D. L., \& Brecht, M. L. (1992). Validity and reliabitity of the Pressure Sore Status Tool. Decubitus, 5(6), 20-28.

Bergin, S. M., Gurr, J. M., Allard, B. P., Holland, E. L., Horsley, M. W., Kamp, M. C., Lazzarini, P. A., Nube, V. L., Sinha, A. K., Warnock, J. T., Alford, J. B., \& Wraight, P. R. (2012). Australian Diabetes Foot Network: management of diabetes-related foot ulceration - a clinical update. Medical Journal of Australia, 197(4), 226-229. https://doi.org/10.5694/mja11.10347

Brasil. (2021). Sistema de Informações sobre Mortalidade - SIM. http://tabnet.datasus.gov.br/cgi/tabcgi.exe?sim/cnv/obt10RJ.def

Cardinelli, C. C., Lopes, L. P. N., Di Piero, K. C., \& Freitas, Z. M. F. (2021). Tools for wound assessment: a scoping review. https://doi.org/ 10.17605/OSF.IO/SREY3

Carville, K., Lewin, G., Newall, N., Haslehurst, P., Michael, R., Santamaria, N., \& Roberts, P. (2007). STAR: a consensus for skin tear classification. Primary Intention, 15(1), 8-25.

Chadwick, P., Edmonds, M., McCardle, J., \& Armstrong, D. (2013). International Best Practice Guidelines: Wound Management in Diabetic Foot Ulcers. Wounds International.

Costa, R. K. de S., Torres, G. de V., Salvetti, M. de G., Azevedo, I. C. de, \& Costa, M. A. T. da. (2015). Instrument for evaluating care given by undergraduate nursing students to people with wounds. Revista Da Escola de Enfermagem Da USP, 49(2), 0317-0325. https://doi.org/10.1590/S0080-623420150000200018

Dantas, D. V., Torres, G. D. V., \& Dantas, R. A. N. (2012). Assistência aos portadores de feridas: caracterização dos protocolos existentes no Brasil. Ciência, Cuidado e Saúde, 10(2). https://doi.org/10.4025/cienccuidsaude.v10i2.8572

Domingues, E. A. R., Carvalho, M. R. F., \& Kaizer, U. A. de O. (2018). Adaptação transcultural de um instrumento de avaliação de feridas. Cogitare Enfermagem, 23(3), e2684. https://doi.org/10.5380/ce.v23i3.54927

EPUAP, NPIAP, \& PPPIA. (2019). Prevention and Treatment of Pressure Ulcers/Injuries: Clinical Practice Guideline (3ª). EPUAP/NPIAP/PPPIA.

Evans, R., Kuhnke, J. L., Burrows, C., Kayssi, A., Labrecque, C., O’Sullivan-Drombolis, D., \& Houghton, P. (2019). Best Practice Recommendations For The Prevention and Management of Venous Leg Ulcers. Canadian Association of Wound Care (Wounds Canada).

Garbuio, D. C., Zamarioli, C. M., Silva, N. C. M. da, Oliveira-Kumakura, A. R. D. S., \& Carvalho, E. C. (2018). Instrumentos para avaliação da cicatrização de lesões de pele: revisão integrativa. Rev. Eletr. Enferm, 20. https://doi.org/10.5216/ree.v20.49425

Günes, U. Y. (2009). A prospective study evaluating the Pressure Ulcer Scale for Healing (PUSH Tool) to assess stage II, stage III, and stage IV pressure ulcers. Ostomy Wound Manage, 55(5), 48-52.

Harris, C., Bates-Jensen, B., Parslow, N., Raizman, R., Singh, M., \& Ketchen, R. (2010). Bates-Jensen Wound Assessment Tool. Journal of Wound, Ostomy \& Continence Nursing, 37(3), 253-259. https://doi.org/10.1097/WON.0b013e3181d73aab

Healey, F. (1997). Classificação das úlceras de pressão II. Nursing, 109, 16-20.

International Diabetes Federation. (2015). IDF Diabetes Atlas (7a.).

Leblanc, K., Baranoski, S., Christensen, D., Langemo, D., Sammon, M. A., Edwards, K., Holloway, S., Gloeckner, M., Williams, A., Sibbald, R. G., \& Regan, M. (2013). International skin tear advisory panel: A tool kit to aid in the prevention, assessment, and treatment of skin tears using a simplified classification system. Advances in Skin and Wound Care, 26(10), 459-476. https://doi.org/10.1097/01.ASW.0000434056.04071.68

Lima, R. V. K. S., Coltro, P. S., \& Farina Júnior, J. A. (2017). Negative pressure therapy for the treatment of complex wounds. Revista Do Colégio Brasileiro de Cirurgiões, 44(1), 81-93. https://doi.org/10.1590/0100-69912017001001

Lipsky, B. A., Berendt, A. R., Cornia, P. B., Pile, J. C., Peters, E. J. G., Armstrong, D. G., Deery, H. G., Embil, J. M., Joseph, W. S., Karchmer, A. W., Pinzur, 
Research, Society and Development, v. 10, n. 11, e144101119246, 2021

(CC BY 4.0) | ISSN 2525-3409 | DOI: http://dx.doi.org/10.33448/rsd-v10i11.19246

M. S., \& Senneville, E. (2012). 2012 Infectious Diseases Society of America Clinical Practice Guideline for the Diagnosis and Treatment of Diabetic Foot Infectionsa. Clinical Infectious Diseases, 54(12), e132-e173. https://doi.org/10.1093/cid/cis346

Mandelbaum, S. H., Di Santis, É. P., \& Mandelbaum, M. H. S. (2003). Cicatrização: conceitos atuais e recursos auxiliares - Parte I. An. Bras. Dermatol., 78(4), 393-408. https://doi.org/10.1590/S0365-05962003000400002

Olczyk, P., Mencner, Ł., \& Komosinska-Vassev, K. (2014). The Role of the Extracellular Matrix Components in Cutaneous Wound Healing. BioMed Research International, 2014, 1-8. https://doi.org/10.1155/2014/747584

Ousey, K., Rogers, A. A., \& Rippon, M. G. (2016). Hydro-responsive wound dressings simplify T.I.M.E. wound management framework. British Journal of Community Nursing, 21(Sup12), S39-S49. https://doi.org/10.12968/bjen.2016.21.Sup12.S39

Peters, M., Godfrey, C., McInerney, P., Munn, Z., Trico, A., \& Khalil, H. (2020). Chapter 11: Scoping Reviews. In JBI Manual for Evidence Synthesis. JBI. https://doi.org/10.46658/JBIMES-20-12

Restrepo-Medrano, J. C., \& Verdú Soriano, J. (2011). Desarrollo de un índice de medida de la evolución hacia la cicatrización de las heridas crónicas. Gerokomos, 22(4), 176-183. https://doi.org/10.4321/S1134-928X2011000400005

Santos, V. L. C. de G., Azevedo, M. A. J., Silva, T. S. da, Carvalho, V. M. J., \& Carvalho, V. F. de. (2005). Adaptação Transcultural Do Pressure Ulcer Scale For Healing (Push) Para A Língua Portuguesa. Rev Latino-Am Enfermagem, 13(3), 305-313.

Silva, C. V. B., Campanili, T., LeBlanc, K., Baranoski, S., \& Santos, V. (2018). Adaptação cultural e validade de conteúdo do ISTAP Skin Tear Classification para o português no Brasil. ESTIMA, Braz. J. Enterostomal Ther, 16, e2618. https://doi.org/10.30886/estima.v16.590_PT

Silveira, I. A., Oliveira, B. G. R. B. de, Souza, P. A. de, Santana, R. F., \& Carvalho, M. R. de. (2020). Cross-cultural adaptation of the Leg Ulcer Measurement Tool for Brazil: nursing methodology research. Revista Brasileira de Enfermagem, 73(4). https://doi.org/10.1590/0034-7167-2018-0944

Strazzieri-Pulido, K. C., Santos, V. L. C. de G., \& Carville, K. (2015). Cultural adaptation, content validity and inter-rater reliability of the "STAR Skin Tear Classification System." Revista Latino-Americana de Enfermagem, 23(1), 155-161. https://doi.org/10.1590/0104-1169.3523.2537

Tchero, H., Kangambega, P., Fluieraru, S., Bekara, F., \& Teot, L. (2019). Management of infected diabetic wound: a scoping review of guidelines F1000Research, 8, 737. https://doi.org/10.12688/f1000research.18978.1

Tricco, A. C., Lillie, E., Zarin, W., O’Brien, K. K., Colquhoun, H., Levac, D., Moher, D., Peters, M. D. J., Horsley, T., Weeks, L., Hempel, S., Akl, E. A., Chang, C., McGowan, J., Stewart, L., Hartling, L., Aldcroft, A., Wilson, M. G., Garritty, C., \& Straus, S. E. (2018). PRISMA extension for scoping reviews (PRISMA-ScR): Checklist and explanation. Annals of Internal Medicine, 169(7), 467-473. https://doi.org/10.7326/M18-0850

Waidman, M. A. P., Rocha, S. C., Correa, J. L., Brischiliari, A., \& Marcon, S. S. (2011). O Cotidiano Do Indivíduo Com Ferida Crônica E Sua Saúde Mental. Texto Contexto Enferm2, 20(4), 691-699.

Woodbury, M. G., Houghton, P. E., Campbell, K. E., \& Keast, D. H. (2004). Development, validity, reliability, and responsiveness of a new leg ulcer measurement tool. Adv Skin Wound Care, 17(4), 187-196. https://www.ncbi.nlm.nih.gov/\%0Apubmed/15360028

Yamada, B. F. A., \& Santos, V. L. C. de G. (2009). Development and Validation of Ferrans \& Powers Quality of Life Index - Wound version*. Rev Esc Enferm USP, 43(Spe), 1033-11. 